\title{
Editorial
}

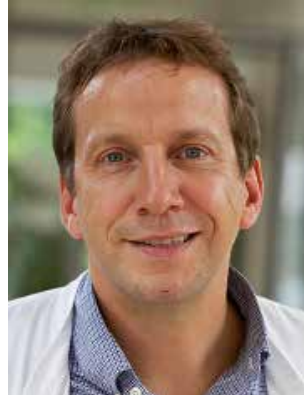

Ein aktueller Beitrag aus der ÄrzteZeitung vom 16. November 2015 trägt die Überschrift „Phytos in Leitlinien unterrepräsentiert“. Eine Kollegin aus der Allgemeinmedizin macht sich hier Luft und weist darauf hin, „Nebenwirkungen und Kosten seien gering und pflanzliche Inhaltsstoffe breit wirksam“. Wie kommt es, dass im Bereich Phytotherapie die medizinische Praxis und deren Abbildung in medizinischen Leitlinien weit auseinanderliegen? Dazu im Folgenden einige Anmerkungen:

Medizinische Leitlinien sind systematisch entwickelte Entscheidungshilfen für Ärzte zu medizinischen Themen. Leitlinien haben den Zweck, die wissenschaftliche Evidenz und die vorhandene Erfahrung zu medizinischen Themen zusammenzufassen, gegensätzliche Standpunkte zu klären und das aktuelle Vorgehen der Wahl zu definieren. Mittlerweile sind Leitlinien zu einem wichtigen Bestandteil des Qualitätsmanagements im Gesundheitswesen geworden, auch wenn sie in erster Linie „nur“ Orientierungshilfen sind, von denen im Einzelfall abgewichen werden kann. Ihr Einfluss ist vielschichtig. Sie dienen einerseits der Orientierung der aktuell im Gesundheitssystem aktiven Ärztinnen und Ärzte. Bedeutend ist weiterhin der Einfluss auf die aktuelle Studenten- und somit die kommende Ärztegeneration, die sich häufig schon nicht mehr an Lehrbüchern, sondern an den aktuellen Leitlinien der AWMF im Internet orientiert. Deutsche Leitlinien zielen dabei nicht nur auf den medizinischen Experten, sie sind häufig in zwei Fassungen erhältlich, eine für den Mediziner und eine kürzere Fassung für den Laien. Außerdem werden die Leitlinien häufig, wenn auch im juristischen Sinne nicht bindend, bei rechtlichen Auseinandersetzungen zur Beurteilung der empfohlenen Versorgung berücksichtigt und können damit relevant für die Berufsausübung werden. Nicht zuletzt werden die Leitlinien zunehmend bei Entscheidungen der Kostenübernahme durch die Krankenkassen und in inhaltlichen Auseinandersetzungen mit dem Medizinischen Dienst als Argumentationsgrundlage genutzt.

\footnotetext{
„Medizinische Leitlinien sind systematisch entwickelte Feststellungen, um die Entscheidungen von Ärzten, Angehörigen anderer Gesundheitsberufe und Patienten über angemessene Gesundheitsversorgung für spezifische klinische Umstände zu unterstützen“ (zitiert nach Lorenz, 1999, AWMF).
}

In Deutschland werden ärztliche Leitlinien von den wissenschaftlichen medizinischen Fachgesellschaften und deren Dachgesellschaft „Arbeitsgemeinschaft der
Wissenschaftlichen Medizinischen Fachgesellschaften (AWMF)“ (http://www.awmf. org) entwickelt und verbreitet.

Medizinische Leitlinien existieren seit den 90er-Jahren des 20. Jahrhunderts. Empfehlungen beinhalteten bis dahin vor allem Expertenmeinungen. Dies entspricht heute nicht mehr dem wissenschaftlichen Anspruch. Zusätzlich zur Darlegung der Erfahrungen und Fachkenntnisse einzelner Experten ist deshalb die systematische Überprüfung und Begutachtung von Studienergebnissen, die zu den spezifischen medizinischen Fragestellungen vorhanden sind, von zentraler Bedeutung. Zudem führt das wachsende Wissen dazu, dass sich Empfehlungen für oder gegen Therapieverfahren ändern können. Aus diesem Grund haben Leitlinien eine kurze „Lebensdauer“ von höchstens 5 Jahren und müssen regelmäßig auf den neuesten Stand gebracht werden. Zur Erstellung einer evidenzbasierten Leitlinie kommen in Deutschland die Vertreter der entsprechenden medizinischen Bereiche und Fachgesellschaften zusammen, um gemeinsam die aktuell existierenden wissenschaftlichen Erkenntnisse zu sammeln und auszuwerten. Der Entstehungsprozess einer solchen Leitlinie nimmt oftmals mehrere Jahre in Anspruch.

Aktuell sind bei der AWMF 416 Leitlinien auf der Entwicklungsstufe S1 verfügbar, die Empfehlungen von individuellen Expertengruppen entsprechen, 202 Leitlinien der Entwicklungsstufe S2, die einer Evidenz- oder einer Konsensus-Basierung entsprechen, und 139 Leitlinien der höchsten Entwicklungsstufe S3, die einer interdisziplinären Evidenz- und Konsensus-Basierung entsprechen, also in der Regel sowohl durch Studien belegt sind als auch eine Mehrheit in den invol- 
vierten Fachgesellschaften der AWMF gefunden haben [1].

Die Situation von Naturheilkunde und Phytotherapie in Hinblick auf öffentliche Forschungsförderung und Gremienarbeit - klassische berufspolitische Machtinstrumente in der Medizin - stellt sich folgendermaßen dar: In Deutschland gibt es, anders als z.B. in der Schweiz, keine öffentlich geförderten Lehrstühle für Naturheilkunde oder Integrative Medizin, obwohl die NHK seit 2003 Pflichtfach des QB12 für das Medizinstudium in Deutschland ist. Die großen öffentlichen Förderer wie DFG und BMBF haben, anders als z. $B$. in den USA, keinen eigenen Forschungsund Förderbereich für Naturheilkunde oder Phytotherapie. Kennzeichnend ist außerdem, dass in wichtigen berufspolitischen Gremien Naturheilkunde und Phytotherapie über Jahre nicht vertreten waren, um dem Bereich Gehör zu verschaffen. So sind in der AWMF, die seit 1995 für die Erstellung der Leitlinien Verantwortung trägt, derzeit 171 wissenschaftliche Fachgesellschaften aus allen Bereichen der Medizin zusammengeschlossen. Bis 2013 war allerdings keine Gesellschaft aus dem Bereich der Naturheilkunde oder Komplementärmedizin Mitglied, was weitreichende Folgen für deren Repräsentation in den AWMF-Leitlinien hatte, die nur durch Zufall von gewogenen Vertretern anderer Fachgesellschaften erfolgte - vor allem aber nicht erfolgte.

Zur Verbesserung dieser aus der Sicht der Phytotherapie so kaum tragbaren Situation sind seit dem Jahr 2011 zwei endscheidende Dinge geschehen:

In Kooperation mit der Gesellschaft für Phytotherapie, der Deutschen Gesellschaft für Naturheilkunde und dem Forum universitärer Arbeitsgruppen für Komplementärmedizin wurde im Jahr 2011, gefördert von der Rut- und KlausBahlsen-Stiftung, die Arbeitsgruppe „Naturheilkunde und Komplementärmedizin in medizinischen Leitlinien" am Lehrstuhl für Naturheilkunde der Universität Duisburg-Essen gegründet. Eine der Hauptaufgaben ist die systematische Aufarbei- tung der vorliegenden Evidenz von Verfahren aus den Bereichen Phytotherapie, Naturheilkunde und Komplementärmedizin als unverzichtbare Grundlage für die Leitlinienarbeit.

Ende des Jahres 2013 erfolgte außerdem durch das umsichtige Engagement von Herrn Prof. Kemper und Frau Prof. Kraft die Aufnahme der Gesellschaft für Phytotherapie in die AWMF und die Benennung einer Person als Leitlinienbeauftragter der GPT. Mit der Aufnahme in die AWMF ist es nun möglich, sich direkt an die Leitlinienkoordinatoren, die Fachgesellschaften und die AWMF zu wenden, wenn eine Überarbeitung oder die Neubearbeitung einer Leitlinie geplant ist, um dann aktiv einen eigenen Fachvertreter mit eigenem Mandat zur Mitarbeit zu entsenden.

Wie wichtig die Arbeit ist zeigen zwei Studien aus unserer Arbeitsgruppe [2, 3]: In 55 der zum Untersuchungszeitpunkt im März 2014 verfügbaren 128 S3-Leitlinien $(42,9 \%)$, mit potenziellen phytotherapeutischen Therapieoptionen, werden pflanzlichen Arzneimittel nicht berücksichtigt. In 40 (31,3\%) der verfügbaren S3-Leitlinien wird Phytotherapie zwar benannt, von den insgesamt 204 Empfehlungen/Kommentaren zu Phytotherapeutika fallen 139 (68,1\%) allerdings negativ aus oder bleiben ergebnisoffen. Hinweise zu Neben- und Wechselwirkungen finden sich zu 70 der 204 Empfehlungen (34,3\%). Es fanden sich also nur in weit weniger als der Hälfte der verfügbaren S3-Leitlinien eine Recherche zu Phytotherapeutika und nur in 17 (13,3\%) der verfügbaren S3-Leitlinien positive Empfehlungen zur Phytotherapie.

In einer zweiten Arbeit wurde untersucht, inwieweit Neben- bzw. Wechselwirkungen von pflanzlichen Arzneimitteln in den verfügbaren S3-Leitlinien diskutiert werden. Im Januar 2015 wurden hierzu die verfügbaren 134 S3-Leitlinien analysiert. Zu diesem Zeitpunkt wiesen 27,6\% ( $n=37)$ insgesamt 194 Statements zur Phytotherapie auf. Hinweise zu Neben- und Wechselwirkungen fanden sich nur bei $28,4 \%$ der Statements $(n=55)$, die bei nur 13,9\% ( $n=27)$ durch Literatur belegt werden. In vielen Fällen zeigten sich unpräzise Begrifflichkeiten sowie eine unzureichende Systematik bei der Suche nach wissenschaftlicher Evidenz für die Beurteilung von Arzneipflanzenzubereitungen.

Die Leitlinienarbeit der Gesellschaft für Phytotherapie kann hier durch die systematische Mitarbeit in den Leitlinien der AWMF einen wichtigen Beitrag zur dringend benötigten Qualitätsverbesserung in diesem Bereich leisten.

Anders als z.B. bei den Monografien des HMPC kann mit ansprechender Forschung unmittelbar Einfluss auf die Gestaltung von medizinischen Leitlinien der Zukunft genommen werden. Notwendige Bedingung hierfür - die „Währung der AWMF“ - ist eine ausreichende Evidenz in qualitativ ansprechenden Studien. Beispiele für aktuelle Forschungsergebnisse geben wir Ihnen in der vorliegenden Ausgabe der ZPT mit Beiträgen zur Wirksamkeit von Kohlblattauflagen bei Gonarthrose, Kümmelölauflagen beim Reizdarmsyndrom und der phytotherapeutischen Behandlungsmöglichkeit der Colitis ulcerosa. Dazu gehört auch eine Übersicht der von Kindern und Jugendlichen in Deutschland häufig verwendeten pflanzlichen Arzneimittel.

\section{Jost Langhorst}

Leitlinienbeauftragter der GPT

\section{Literatur}

1 http://www.awmf.org/leitlinien/aktuelle-leitlinien.html

2 Klose P, Kraft K, Cramer H, Lauche R, Dobos G, Langhorst J. Phytotherapie in den medizinischen S3-Leitlinien der Arbeitsgemeinschaft der Wissenschaftlichen Medizinischen Fachgesellschaften - eine systematische Übersichtsarbeit. Forsch Komplementärmed Klass Naturheilkd 2014; 21: 388-400

3 Klose P, Kraft K, Cramer H, Lauche R, Dobos G, Langhorst J. Berücksichtigung von Wechselund Nebenwirkungen bei pflanzlichen Arzneimitteln in deutschen S3-Leitlinien der AWMF. Forsch Komplementärmed Klass Naturheilkd 2016 (im Druck) 